101 BIOMECHANICAL RISKS OF KNEE INJURY IN MIXED MARTIAL ARTS: A VIDEO-BASED NOTATION ANALYSIS

${ }^{1,3}$ Kam Ming Mok, ${ }^{1}$ Ivan YH Lau, ${ }^{2}$ Lobo Louie, 'Patrick Shu Hang Yung. 'Department of Orthopaedics and Traumatology, Faculty of Medicine, The Chinese University of Hong Kong, Hong Kong, Hong Kong (China); ${ }^{2}$ The Department of Sport and Physical Education, Hong Kong Baptist University, Hong Kong, Hong Kong (China); ${ }^{3}$ Office of Student Affairs, Lingnan University, Hong Kong, Hong Kong (China)

\subsection{6/bjsports-2021-IOC.94}

Background Incidence rate of knee injury in mixed martial arts (MMA) is high. MMA is a high-intensity sport that requires constant angle and level change. A majority of the combined movements overlap with biomechanics that have been suggested to be associated with increased risk of knee injury.

Objective To identify the frequency of occurrence of the highrisk movements, i.e. dynamic valgus, during MMA competitions.

Design Video-based notation analysis.

Setting The analysed movements have been separated into two categories, Gross Biomechanical Risks and Detailed Biomechanical Risks. High-intensity movements were analysed in slow motion to ensure accuracy. Three fights were re-watched after all 29 fights as quality control to access the reliability of measurement.

Patients (or Participants) 29 MMA fights.

Interventions (or Assessment of Risk Factors) Documenting all visible high-risk knee injury movements occurred in the fights.

Main Outcome Measurements The frequency of occurrence of the high-risk movements, i.e. dynamic valgus, during MMA competitions. In addition, other gross biomechanics were recorded.

Results On average, an MMA athlete experienced 31 times of knee valgus motion with explosive force drag during a match. The side affected was highly subjective to the stance of the fighter. The rear leg was most likely to go through knee valgus motion.

Conclusions The result showed high-risk knee motions are common in MMA. Based on the data, knee valgus on the rear leg is of interest for future injury prevention focus. The data collected in this study can be used as the backbone for further investigation on the possible ways for knee injury prevention.

\section{TOP-LEVEL KARATE ATHLETES: ARE THEY IMPLEMENTING PREVENTATIVE INJURY MEASURES?}

${ }^{2}$ Montassar Tabben, ${ }^{1}$ Dusana Cierna, ${ }^{4}$ Laura Perez Martin, ${ }^{3}$ Rafael Arriaza. ${ }^{1}$ Physical Education and Sports School, Comenius University, Bratislava, Slovakia; ${ }^{2}$ Aspetar Orthopedic and Sports Medicine Hospital, Doha, Qatar; ${ }^{3}$ Instituto Médico Arriaza y Asociados, Coruña, Spain; ${ }^{4}$ University of Alcalá, Madrid, Spain

\subsection{6/bjsports-2021-IOC.95}

Background The current status of injury prevention implementation in top-level karate is unknown.

Objective To examine the current perceptions and practices of top-level karate athletes concerning risk-factors and injury prevention implementation.

Design Cross-sectional study based on face-to-face surveys.

Setting During the official medical meeting of the karate World Senior Championships (WSC) Madrid, 2018, the Chairman of the Word Karate Federation Medical Commission presented the project and invited the 131 team doctors to inform their athletes about the survey. Only athletes that accepted the invitation and speaking English, French, Slovak, Polish, Czecho and/or Spanish were involved in this study.

Patients (or Participants) Out of a total of 1117 athletes (140 countries/teams) that participated in the WSC, 137 (50 countries/teams) have completed the questionnaires (399 athletes didn't speak any of the mentioned languages, 201 athletes didn't volunteer and 380 couldn't be reached)

Interventions (or Assessment of Risk Factors) The survey was constructed face to face and consisted of 15 questions.

Main Outcome Measurements The current perceptions and practices of top-level karate athletes concerning risk factors and injury prevention implementation.

Results Only $44.7 \%$ of the participant athletes have reported that their teams have conducted any preventive measures to reduce injury risk. Kumite athletes (51.2\%) were more likely to practice injury prevention compared to kata athletes $(25 \%$; $\mathrm{P}=0.016)$. Athletes who received preliminary advices about injury prevention (58\%) were more likely to practice it, compared to who did not receive any advice $(20.5 \% ; \mathrm{P}<0.001)$. Athletes who have a fitness coach (part time or full time) were practicing more injury prevention strategies $(66.7 \%$ and $51.4 \%$, respectively) compared to athletes who do not have a fitness coach $(35 \% ; \mathrm{P}=0.031)$.

Conclusions Injury prevention advices and the presence of a fitness coach are associated with an increase in the injury prevention practice. Action plans regarding injury prevention education should be implemented.

\section{THE EPIDEMIOLOGY OF TRAUMATIC BRAIN INJURIES WITHIN USA FENCING, 2015-2020: PREVENTION, CARE, AND RETURN TO PLAY CONSIDERATIONS}

${ }^{1}$ Adam Thompson, ${ }^{2}$ Jeremy Summers, ${ }^{3}$ Alan Freedman. 'Indiana Wesleyan University, Marion, IN, USA; '2 USA Fencing, Colorado Springs, CO, USA; ${ }^{3}$ University of Illinois Springfield, Springfield, IL, USA

\subsection{6/bjsports-2021-IOC.96}

Background Mild traumatic brain injury (mTBI) is an increasing sports-related and public health concern affecting millions annually. While competitive fencing has been identified as a safe sport with a low occurrence of sport related injury, prevention, care, and return to play considerations from a mTBI is a persisting issue due to sport related factors.

Objective To identify incidence and prevalence of mTBI over 5 competitive fencing seasons through a Sport Event Medical Encounter System (SEMES).

Design Documented mTBI sustained at USA Fencing sponsored domestic events and individual and national team involvement at international events were analyzed from the 2015-2020 competitive seasons.

Setting All SEMES mTBI data collected at USA Fencing sponsored domestic fencing events involving Y14 through veteran competitors as well as individual and national team involvement at international events were included in the analysis.

Patients (or Participants) Inclusion criteria involved both genders within the competition categories of Y14 through veteran divisions. Among 98,000 fencing competitors, 75 sustained a mTBI requiring medical withdrawal.

Interventions (or Assessment of Risk Factors) USA Fencing has an annual competitive growth of $5 \%$ over the past five 
seasons. Increased staffing of credentialed sports medicine clinicians at domestic and international events to recognize and document mTBI.

Main Outcome Measurements Improved documentation of mTBI to influence prevention, care, and return to play interventions.

Results Data identified two primary mechanisms of injury leading to diagnosed mTBI: indirect head trauma from a blow to the mask from an opponent's weapon guard and falling backwards onto the strip causing direct posterior trauma to the head. The acceleration-deceleration mechanism of injury (whiplash) was also a component in both of these identified injury mechanisms.

Conclusions Increased clinician staffing at domestic and international events has directly correlated with an increase in documentation and overall mTBI prevention, diagnosis, care, and return to play considerations.

\section{CURRENT PRACTICE FOR SAFE RETURN-TO-PLAY AFTER LATERAL ANKLE SPRAIN: A SURVEY AMONG FRENCH- SPEAKING PHYSICIANS}

${ }^{1}$ Aude Aguilaniu, ${ }^{1}$ Jean-Louis Croisier, ${ }^{3}$ Cédric Schwartz, ${ }^{2}$ Nadia Dardenne, ${ }^{5}$ Pieter D'Hooghe, ${ }^{4}$ Yanis Zahraoui, ${ }^{1}$ Romain Collin, ${ }^{1}$ Jean-François Kaux, ${ }^{1,6}$ Géraldine Martens. 'Department of Sport Sciences of University of Liège, Liège, Belgium; ${ }^{2}$ Département of public health of science of University of Liège, Liège, Belgium; ${ }^{3}$ Laboratory of Human Motion Analysis, Faculty of Applied Sciences, University of Liege, Liège, Belgium; ${ }^{4}$ Physiotherapist, Liège, Belgium; ${ }^{5}$ Chief of Surgery at Aspetar Hospital, Doha, Qatar; ${ }^{6}$ ReFORM IOC Research Centre, Liège, Belgium

\subsection{6/bjsports-2021-IOC.97}

Background The incidence of lateral ankle sprain (LAS) in sports and risk of LAS re-injury is high.

Objective The purpose of this study was to see how physicians use existing recommendations in the literature to decide a safe return-to-play (RTP) after a LAS. We hypothesize that physicians are using the published recommendations to make this RTP decision.

Design An online survey was designed to identify clinical practice patterns to decide a RTP decision after a LAS.

Setting The physicians were contacted by e-mail containing an electronic link for survey access between January and February 2019. They were instructed to answer all questions that related to their clinical practice of patients after a LAS.

Patients (or Participants) 109 physicians answered the entire survey. Among the 109 physicians, 59 had a sport education and 50 had no specific sport education.

Interventions (or Assessment of Risk Factors) Physicians selected the criteria and details on the assessment they used in daily practice for RTP decision after a LAS.

Main Outcome Measurements Physicians should select a maximum of 5 items among the 9 suggested: 'pain, swelling, ankle range of motion, ankle muscle strength, feeling of instability, proprioception, ability to do functional tasks and other criteria'.

Results Pain, functional and instability criteria are the most selected by overall physicians. Sport physicians (94\%) selected significantly more the functional criterion than physicians without sport specialty $(71 \%) \mathrm{p}=0.0064, \mathrm{OR}=0.095$ $[0,017 ; 0,515]$. Sport physicians (64\%) selected significantly more a quantitative value to assess the pain (visual scale) than physicians without sport education $(41 \%) \mathrm{p}=0.03$. Few physicians use a questionnaire to assess the instability (less than $5 \%$ ).

Conclusions In daily practice, physicians with sport education are more aware of functional test guidelines to assess a LAS in RTP context. Few physicians use quantitative tools and measures to decide a safe RTP after a LAS.

\section{BALANCE CONTROL IN CHRONIC ANKLE INSTABILITY AND HEALTHY SUBJECTS: TRADITIONAL ANALYSIS OF CENTRE OF PRESSURE DISPLACEMENT AND NONLINEAR ANALYSIS OF ITS VARIABILITY}

\begin{abstract}
${ }^{1,2}$ José Esteves, ${ }^{2}$ Ricardo Dinis, ${ }^{3}$ Orlando Fernandes, ${ }^{4}$ Maria António Castro, ${ }^{2,5}$ Raul Oliveira, 2,5Pedro Pezarat Correia. ${ }^{1}$ Escola Superior de Saúde do Alcoitão, Lisboa, Portugal: ${ }^{2}$ Neuromuscular Research Lab, Faculdade de Motricidade Humana - Universidade de Lisboa, Lisboa, Portugal; ${ }^{3}$ Departamento de Desporto e Saúde - Universidade de Évora, Évora, Portugal; ${ }^{4}$ Escola Superior de Tecnologia da Saúde de Coimbra, Coimbra, Portugal; ${ }^{5}$ CIPER, Faculdade de Motricidade Humana - Universidade de Lisboa, Lisboa, Portugal
\end{abstract}

\subsection{6/bjsports-2021-IOC.98}

Background Many causes of chronic ankle instability (CAI) have been postulated and include deficits in proprioception, impaired neuromuscular firing patterns, reduced balance and postural control. Balance control can be studied with the traditional analysis of centre of pressure (CoP) displacement and nonlinear analysis of its variability.

Objective To compare balance control behaviour in CAI and healthy subjects, using the traditional linear and nonlinear variables for CoP displacement.

Design Comparative observational study.

Setting Laboratory.

Patients (or Participants) Students from 2 universities in Lisbon, who volunteered to participate in this study: 16 subjects with chronic ankle instability and 20 healthy subjects. Selection was based on the International Ankle Consortium (IAS) selection criteria position statement.

Interventions (or Assessment of Risk Factors) The independent variable was the presence of CAI according to IAS selection criteria.

Main Outcome Measurements CoP displacement was measured on a force plate during single leg stance for 60 seconds, on stable and unstable surfaces. Balance control was measured through traditional variables of CoP displacement: total displacement; medio-lateral and antero-posterior displacement; amplitude of medio-lateral and antero-posterior displacement. Variability of CoP displacement measured with nonlinear variables: sample entropy of medio-lateral and antero-posterior displacement; correlation dimension of medio-lateral and anteroposterior displacement.

Results On stable surface, no differences between groups for all the traditional variables were found but correlation dimension of CoP medio-lateral displacement had lower values on CAI group with statistical significance $(\mathrm{p}<0,05)$. On unstable surface no differences were found neither with traditional variables or variability nonlinear analysis.

Conclusions Besides no differences on most of variables, lower values of the correlated dimension of CoP displacement during one leg stance on a stable surface in CAI subjects may indicate a balance control system with less variability to adapt to the environment and the task demands. More studies are needed to continue the investigation about the balance control related to CAI. 